EPJ Web of Conferences 13, 07003 (2011)

DOI: $10.1051 /$ epjconf/20111307003

(C) Owned by the authors, published by EDP Sciences, 2011

\title{
Quark Number Scaling in Fluid Dynamics and Hadronization via Quarkyonic Matter
}

\author{
L.P. Csernai ${ }^{1,2,3}$, Y. Cheng ${ }^{1}$, Sz. Horvat ${ }^{1}$, I. Mishustin ${ }^{3}$, and S. Zschocke ${ }^{4}$ \\ 1 Department of Physics and Technology University of Bergen, 5007 Bergen, Norway \\ 2 MTA-KFKI Research Inst. for Particle Physics and Nuclear Physics, 1525 Budapest, Hungary \\ ${ }^{3}$ Frankfurt Institute for Advanced Studies (FIAS), 60438 Frankfurt am Main, Germany \\ 4 TU Dresden, Institut für Planetare Geodäsie, Lohrmann-Observatorium, D-01062 Dresden, Germany
}

\begin{abstract}
NCQ scaling of elliptic flow is studied in a non-equilibrium hadronization and freeze-out model from ideal, deconfined and chirally symmetric Quark Gluon Plasma (QGP), to final non-interacting hadrons. In this transition the quarks gain constituent quark mass while the background Bag-field breaks up. The constituent quarks then recombine into simplified hadron states, while chemical, thermal and flow equilibrium break down. Then the resulting temperatures and flow velocities of baryons and mesons will be different. In a simplified model, we reproduce the constituent quark number scaling.
\end{abstract}

\section{Introduction}

The elliptic flow is characterized by the second coefficient of the Fourier expansion of the momentum distribution, $v_{2}$. It was found [1] that the $v_{2}$ parameter as a function of the transverse momentum, $p_{\perp}$, scales with the number of constituent quarks, $n_{c q}$, in the detected hadron: if the $v_{2}\left(p_{\perp}\right)$ curves are re-scaled according to the constituent quark number (NCQ), so $v_{2} / n_{c q}$ is plotted as function of $p_{\perp} / n_{c q}$ for each type of hadron, the curves will coincide. Later results showed that the scaling is more precise if $v_{2}$ is plotted versus the transverse kinetic energy, $E_{\perp}=$ $\sqrt{m^{2}+p_{\perp}^{2}}-m$

This experimentally found NCQ scaling law indicates that the elliptic flow develops before the quarks recombine into hadrons. Therefore, the $v_{2}$ can provide information about the state of the QGP.

The scaling of the flow was studied in Fluid Dynamical (FD) models, where very frequently a close to linear $v_{2}\left(p_{\perp}\right)$ dependence was predicted, which than reproduces the NCQ scaling[2]. This, however, was a consequence of the choice of a constant freze out (FO) temperature and this is not a consequence of the FD approach. If different fluid elements have different temperatures at FO, this type of scaling does not occur. This can be demonstrated in the simple three source model where we have a hotter central source. See Figure 1.

The FD model describes the dynamical development of the QGP from the (already thermalized) initial state until the break-down of the equilibrium, where 1st the chemical equilibrium among quarks and antiquarks ceases. Energy and momentum conservation and the requirement of non-decreasing entropy is enforced in the transition, from the ideal QGP state to the state where quark and anti-quark

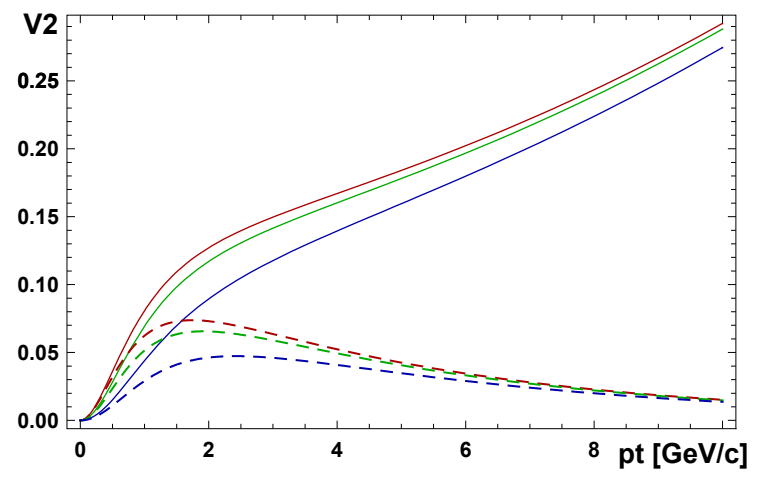

Fig. 1. (color online) $v_{2}$ versus $p_{\perp}$ from the three source model, with two side sources of mass number, $A_{s}=50$, velocity, $\pm v_{x}$, where $v_{x}=0.2$ and temperature, $T_{s}=100 \mathrm{MeV}$. The 3rd central static source has mass number, $A_{c}=100$. Above and below the critical temperature of the central source $T_{c r i t}=T_{s} /\left(\gamma_{x}-u_{x}\right)$ the elliptic flow tends to 0 and 1 respectively. Two sets of curves are shown for the indicated particle masses $(m=0.15,0.5,1$ $\mathrm{GeV}$, [red, green, blue] ) from up down, first with temperature $T_{c}$ $2 \%$-below and then $2 \%$-above the critical one. The two groups of curves tending to 1 and 0 respectively with increasing $p_{\perp}$.

numbers are frozen out. As a consequence, the mass change of the quarks starts in the initial QGP and we estimate the final boundary where the FD stage of the evolution ends, with given constituent quark masses.

In this model, a gas of quarks and anti-quarks expands in a background field of energy density, $B$. Initially this B-field includes the energy of the deconfined perturbative vacuum and of the gluon fields. As the system expands the deconfinement starts and the average B decreases. When

This is an Open Access article distributed under the terms of the Creative Commons Attribution-Noncommercial License 3.0, which permits unrestricted use, distribution, and reproduction in any noncommercial medium, provided the original work is properly cited. 
chiral symmetry breaking starts and the quarks gain mass. Their mass is calculated as function of the temperature and density. This process can be considered as a simple representation of the chiral symmetry breaking and deconfinement in a dynamical transition crossing the Quarkyonic phase [4].

The point when the quarks recombine into hadrons is determined from the condition that the average hadron energy is equivalent to $1.0-1.1 \mathrm{GeV}$, as found from the systematics of experimental data [3] At recombination, the thermal and flow equilibrium between particles is broken. The $v_{2}$ parameter is determined using two- and three-source models of the elliptic flow and the particle distributions obtained from the hadronization model.

\section{Change of Constituent Quark Mass}

To present our arguments we consider the Nambu-JonaLasinio model (NJL) which is motivated by QCD and is basically a purely quark-quark-interaction theory.

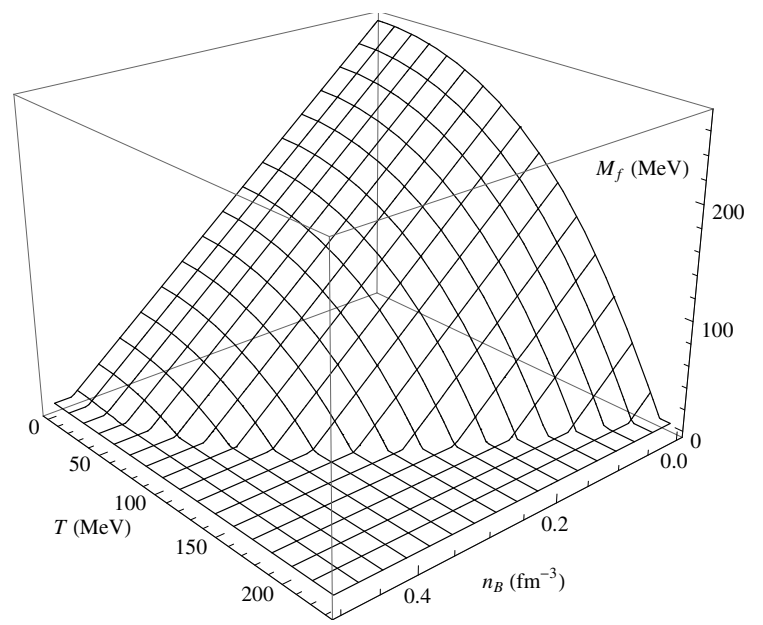

Fig. 2. Temperature and density dependence of the constituent quark mass $M_{f}$ according to Eq. (7).

First, let us consider the constituent quark mass in vacuum. For three flavours $f$, i.e. for $S U_{f}(3)$, the constituent quark masses in vacuum, $M_{f}^{0}=M_{f}\left(n_{B}=0, T=0\right)$ where $f=u, d, s$, are given by $[5,6]$

$$
\begin{aligned}
& M_{u}^{0}=m_{u}-2 G_{S}\langle\bar{u} u\rangle_{0}-G_{D}\langle\bar{d} d\rangle_{0}\langle\bar{s} s\rangle_{0}, \\
& M_{d}^{0}=m_{d}-2 G_{S}\langle\bar{d} d\rangle_{0}-G_{D}\langle\bar{u} u\rangle_{0}\langle\bar{s} s\rangle_{0}, \\
& M_{s}^{0}=m_{s}-2 G_{S}\langle\bar{s} s\rangle_{0}-G_{D}\langle\bar{u} u\rangle_{0}\langle\bar{d} d\rangle_{0},
\end{aligned}
$$

where $m_{u}=5 \mathrm{MeV}, m_{d}=9 \mathrm{MeV}$ and $m_{s}=130 \mathrm{MeV}$ are the current quark masses of the u-quark, d-quark and squark, respectively, and $G_{S}$ is the coupling constant of the scalar-current interaction, and $G_{D}$ is the coupling constant of the determinantal flavour-mixing term (determinant in flavour space). According to Eqs. (1) - (3), the constituent quark masses are related to the chiral condensates in vacuum. Typical values of the chiral condensates in vacuum are given by, cf. Refs. [7,8]:

$$
\begin{aligned}
\langle\bar{q} q\rangle_{0} & =\langle\bar{u} u\rangle_{0}=\langle\bar{d} d\rangle_{0}=-(0.225 \mathrm{GeV})^{3}, \\
\langle\bar{s} s\rangle_{0} & =0.7\langle\bar{u} u\rangle_{0} .
\end{aligned}
$$

Typical values for the coupling constants are $G_{S}=(15-$ 20) $\mathrm{GeV}^{-2}, G_{V} \simeq 0.5 G_{S}$ and $G_{D}=-(160-240) \mathrm{GeV}^{-5}$ $[5,6]$.

Let us now consider the case of quarks in a hot medium and dense medium, at early times of FD evolution. At this initial stage we assume to have two flavours only as we assume that in the initial FD state the flavour balance is not established yet. Then, relations (1) - (3) for $S U_{f}(2)$ simplify to

$$
M_{f}=m_{f}-2 G_{S}\langle\bar{q} q\rangle_{n_{B}, T} .
$$

The suffix $n_{B}, T$ at chiral condensate denotes the average over hadron and meson states [7,9]. In the limit of high densities and temperatures the constituent quark mass, $M_{f}$, approaches the current quark mass, $m_{f}$.

Here, in order to determine the density and temperature dependence of the chiral condensate we follow the arguments of Ref. [6-8], where the first leading terms of $\langle\bar{q} q\rangle_{n_{B}, T}$ in the low-density low-temperature expansion have been obtained[9] in terms of $\langle\bar{q} q\rangle_{0}$ and the temperature and baryon density, $n_{B}=\sum_{f=u, d}\left(n_{f}-\bar{n}_{f}\right) / 3$. Thus, we obtained the expression for the in-medium mass of constituentquarks $q$ (either $u$ or $d$ ):

$$
\begin{aligned}
& M_{f}=m_{f}-2 G_{s}\langle\bar{q} q\rangle_{0} \\
& \times\left(1-\frac{3 \sigma_{q}}{f_{\pi}^{2} m_{\pi}^{2}} n_{B}-\frac{T^{2}}{8 f_{\pi}^{2}}-\frac{T^{4}}{384 f_{\pi}^{4}}-\frac{T^{6}}{288 f_{\pi}^{6}} \ln \frac{\Lambda_{q}}{T}\right),
\end{aligned}
$$

where for the logarithmic scale we take $\Lambda_{q} \simeq 300 \mathrm{MeV}$, the pion mass in vacuum is $m_{\pi}=138 \mathrm{MeV}$ and the pion decay constant in vacuum is $f_{\pi}=93 \mathrm{MeV}$. The numerical value of the quark-sigma-term is $\sigma_{q}=15 \mathrm{MeV}$ (see e.g. Ref. [6]), which is three times smaller than the nucleonsigma-term $\sigma_{N}=45 \mathrm{MeV}$.

The temperature and density dependence of the constituent quark mass is plotted in Fig. 2. According to Eq. (7), for sufficiently high temperatures and densities the constituent quark mass will coincide with the current-quarkmass $m_{f}$.

\section{Non-equilibrium Expansion}

The total energy of the bag in the centre-of-mass frame of the colliding nuclei is given by the volume integral of the "00" component of the energy momentum tensor of each cell $i, T_{i}^{\mu \nu}=(e+P) u^{\mu} u^{v}-P g^{\mu \nu}$. Considering that our EoS is given as a sum of the energy and pressure of the ideal quark parton gas, $e^{i}, P^{i}$, and the bag energy density, $B$, as $e=e^{i}+B$ and $P=P^{i}-B$, the "00" component becomes 
$T_{i}^{00}=\left(e^{i}+P^{i}\right)\left(\gamma^{i}\right)^{2}-\left(P^{i}-B\right)$. Thus the total energy of all cells of the bag in the centre-of-mass frame of the colliding nuclei is given by

$$
E_{\text {total }}=\sum_{i=1}^{N} V^{i}\left(\gamma^{i}\right)^{2}\left(e^{i}+P^{i}\right)-\sum_{i=1}^{N} V^{i} P^{i}+V B,
$$

where the sum runs over the number of all cells of the bag. Here, we assume a uniform bag-field energy density, $B$, over the whole volume, $V_{C M}$, which may depend on density and temperature of the bag, thus on the time of the evolution of the fireball. We have used the notation $e^{i}$ for the invariant scalar, rest-frame energy density, and $P^{i}$ for the pressure, $V^{i}$ is the volume of cell $i$ in the centre-ofmass frame, and $\gamma_{i}=1 / \sqrt{1-\mathbf{v}_{i}^{2}}$ where $\mathbf{v}_{i}$ is the 3 -velocity of cell $i$.

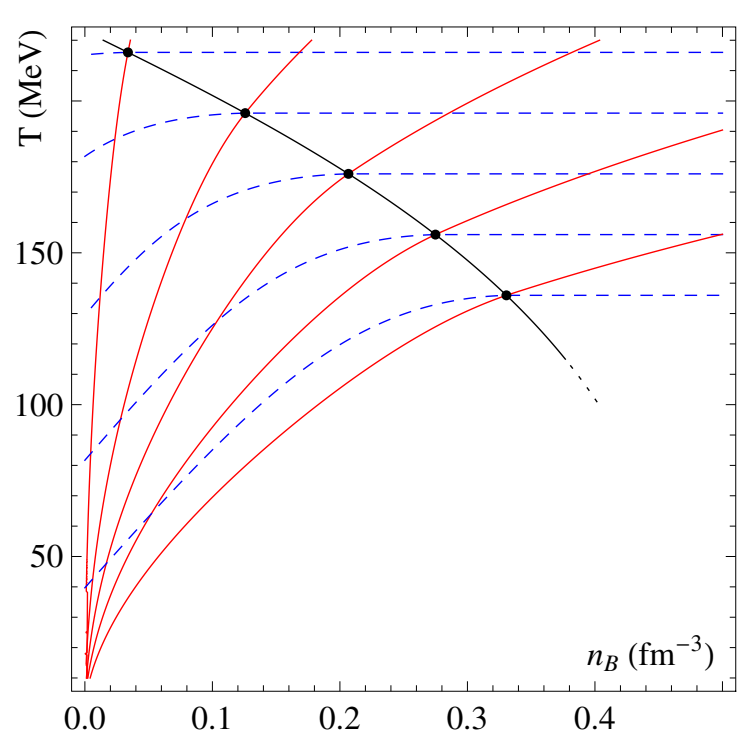

Fig. 3. (color online) Expansion of the gas of quarks and antiquarks. The thick (black) solid line is calculated assuming the quark masses to be equal to the current quark mass, that means it is identical with the boundary curve in Fig. 2. Beyond that curve, at higher temperatures and densities, we take $M_{f}=m_{f}$. The crossing points on the thick (black) solid line indicate the initial temperature and initial baryonic density for the non-equilibrium expansion. From here each thin (red and blue) curve shows the path of adiabatic and iso-ergic expansion respectively. The adiabatic expansion (red curves) cool faster and thus more energy is converted into flow. The trajectories of the iso-ergic dissipative expansion (blue lines) are plotted for comparison. For these the temperature falls slower.

In order to determine the rest-frame energy or proper energy of the cells, we assume a Jüttner distribution for the quarks and anti-quarks in each cell. This makes it possible to evaluate the quantities, $e^{i}, P^{i}$, in therms of cell temperature and chemical potential assuming still chemical equi- librium. Similarly the total momentum and total entropy can also be evaluated

$$
\begin{aligned}
0=\boldsymbol{p}_{\text {total }} & =\sum_{i=1}^{N} V^{i}\left(\gamma^{i}\right)^{2}\left(e^{i}+P^{i}\right) \boldsymbol{v}_{i}, \\
S_{\text {total }} & =\sum_{i=1}^{N} V^{i} \gamma^{i} s^{i} .
\end{aligned}
$$

In early stages of FO, when chemical equilibrium still exist we can get in terms of a single chemical potential, $\mu_{q}=\mu_{B} / 3$ and $\mu_{\bar{q}}=-\mu_{B} / 3$ the quark, anti-quark and total and net baryon densities. In our model we assume this until the point when the quarks start to acquire mass, see the corresponding boundary-line on the $[T, n]$ plane in Figure 2. At this boundary first the chemical equilibrium (as well as chiral symmetry) break and the quark and antiquark numbers are fixed separately! Thus, during the subsequent, non-equilibrium expansion a unique chemical potential does not determine the quark densities, to the contrary, the given fixed quark and anti-quark numbers (and the corresponding decreasing densities) will provide different chemical potentials for quarks and antiquarks $\left(\mu_{q} \neq\right.$ $\left.-\mu_{\bar{q}}\right)$ !

The reaction until this stage can be described well in the FD model, which assumes local equilibrium. The QGP is a nearly perfect fluid, thus a perfect FD method is adequate, like the relativistic Particle in Cell (PIC) method. One has, nevertheless, to remember that some viscosity is needed for the stability of the flow, and on theoretical grounds there must also be a minimal viscosity[10]. In the PIC method, line in most perfect numerical methods this minimal viscosity ensured by the numerical viscosity. The numerical viscosity of the PIC method is recently studied in reference [11].

There are indications $[13,14]$ that freeze out and hadronization happen rapidly and simultaneously from supercooled QGP. This happens from the point when the quarks start to acquire mass, until we reach the empirical hadron freeze out line [3]. We estimated this boundary on $\left[T, \mu_{B}\right]-$ plane in the Boltzmann-Jüttner gas approach, see Fig. 2 in ref. [12]. Let us assume adiabatic expansion between these two boundaries.

Using the condition that the total entropy is constant, i.e. the entropy density decreases as $s(T, V)=s_{q}(T, V)+$ $s_{\bar{q}}(T, V)=V_{0} / V s_{0}$, the expansion trajectories on the $\left[n_{B}, T\right]$ plane can be calculated numerically. These trajectories are plotted in Figure 3.

\subsection{Recombination into hadrons}

Due to confining forces, finally quarks will recombine into hadrons. This happens rapidly, out of equilibrium, at the point of recombination when the average energy per hadron (including the background field) decreases to $E_{H} / N_{H}=1.2$ $\mathrm{GeV}$. This value is still above the values of $(1.0-1.1) \mathrm{GeV}$ obtained by Cleymans et al. [3]. This corresponds nearly to 
the energy per hadron of the empirically observed freezeout. The endpoints of the expansion curves where the recombination happens are shown in Fig. 4. At the same time the rest of the energy is redistributed so that different hadron species will have different thermal and flow energies leading to different temperature parameters and flow velocity parameters. These parameters are actually not the intensive thermodynamical parameters, which only exist in equilibrium, these only parametrize the non-equilibrated distributions of the different hadron species.

Hadrons are assumed to have a mass that is the sum of the masses of their constituent quarks at the FO, i.e.

$$
\begin{aligned}
& M_{b}=M_{\bar{b}}=3 M_{f}\left(n_{B}^{\mathrm{FO}}, T^{\mathrm{FO}}\right), \\
& M_{m}=2 M_{f}\left(n_{B}^{\mathrm{FO}}, T^{\mathrm{FO}}\right) .
\end{aligned}
$$

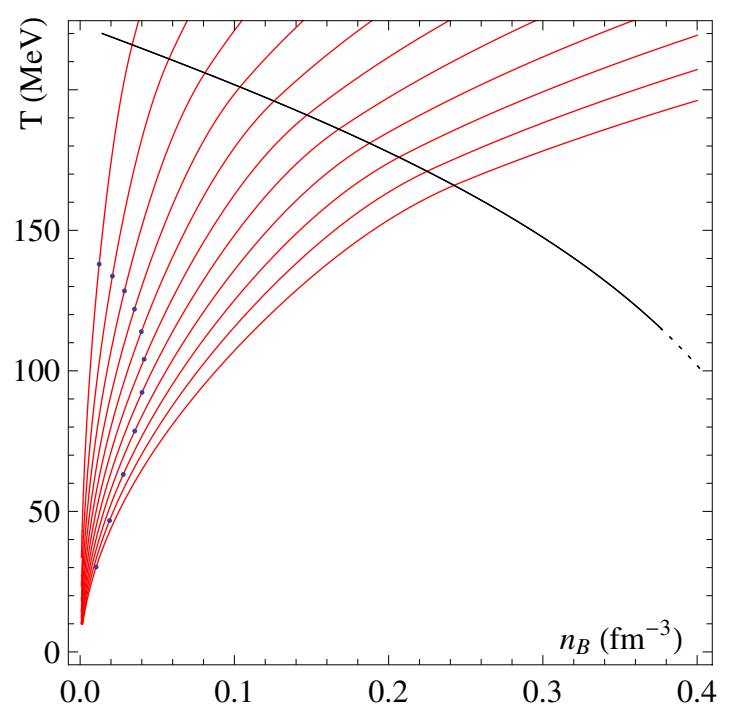

Fig. 4. (color online) A series of curves of adiabatic expansion of the gas of quarks and anti-quarks. The crossing points on the adiabatic expansion curves indicate where the rapid freeze-out and hadronization happens. These points are determined based on the condition that the energy of the system, including the background field, divided by the estimated number of hadrons reaches 1.2 GeV hadron, still above the observed FO energy. The solid (black) line is the same as in Figure 3.

Other differences between the various hadron species are disregarded. Most of the anti-quarks will pair with quarks to form mesons, but a small fraction, $a$, will form antibaryons. This ratio, $a$, can be estimated based on the recombination rates given in [16]. Thus the baryon, antibaryon and meson densities $\left(n_{b}, n_{\bar{b}}\right.$, and $\left.n_{m}\right)$ are calculated from the quark and anti-quark densities $\left(n_{q}\right.$ and $\left.n_{\bar{q}}\right)$. See reference [12].

The parameters of the distribution after recombination are calculated from the condition of energy conservation: the thermal energy of each hadron type will be equal to the energy of their constituent quarks, $E_{M}^{t h}=E_{B}^{t h}$. Due to the different masses of baryons and mesons, their temperature parameters will be different. The temperature ratio $T_{b} / T_{m}$ will correspond to the mass ratio $M_{b} / M_{m}=3 / 2$. The distributions of baryons and mesons for a calculation done with initial state $n_{B 0}=0.21 \mathrm{fm}^{-3}$ and $T_{\mathrm{q} 0}=176 \mathrm{MeV}$. The resulting final baryon and meson temperatures become $T_{b}=228 \mathrm{MeV}$ and $T_{m}=152 \mathrm{MeV}$ and $T_{b} / T_{m}=3 / 2$, while the final constituent quark mass $308 \mathrm{MeV}$.

The resulting scaled $p_{\perp}$ and $E_{\perp}$ distributions become identical under this condition, however this is not enough to reproduce the NCQ scaling of $v_{2}\left(p_{\perp}\right)$ indicating that the recombination influences the flow velocities of the final hadrons. This concept was already pointed out in ref. [16] based on the properties of the collision integral. It is important to point out that the transport theoretical treatment and the collision integral are applicable also at situations when the local equilibrium has ceased to exist.

\section{The Elliptic Flow}

The elliptic flow parameter, $v_{2}$, can be calculated from the final, post FO distribution by the Cooper-Frye formula. Assuming an isochronous FO hypersurface, we obtain simple expressions for final measurables just as in ref. [2].

As a first approximation, the $v_{2}$ parameter was calculated in a two source model, the sources moving in opposite directions with the same velocity. The baryons and mesons were given different flow energies, such that the ratio of flow energy per quark is $\left(F E_{b} / n_{c q}\right) /\left(F E_{m} / n_{c q}\right)=$ $3 / 2$. This leads to different flow velocities for baryons and mesons $v_{M}=0,21 c \neq v_{B}=0.26 c$, and reproduces the NCQ scaling of the elliptic flow parameter, up to $p_{\perp}=400$ $\mathrm{MeV}$. By this point of the evolution, all energy in the background field is exhausted and the internal, excitation and random kinetic energies of the hadrons reach the FO value, $(1.0-1.1) \mathrm{GeV}$.

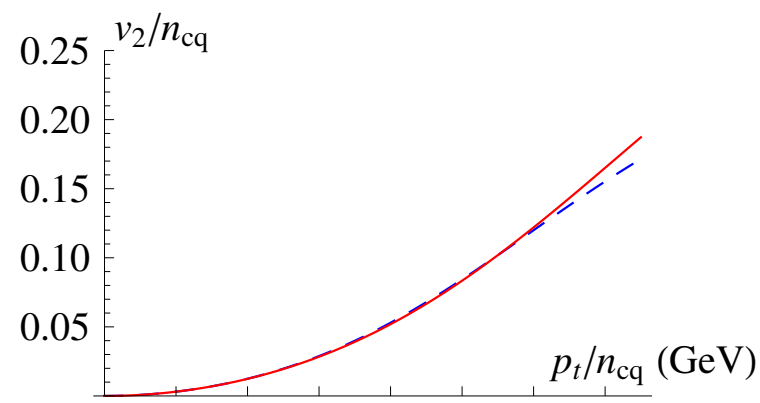

$\begin{array}{llllllll}0 . & 0.2 & 0.4 & 0.6 & 0.8 & 1 . & 1.2 & 1.4\end{array}$

Fig. 5. (color online) The re-scaled elliptic flow parameter, $v_{2} / n_{c q}$, as a function of $p_{\perp} / n_{c q}$, calculated from the three-source model. The dashed (black) curve represents the baryons while the solid (red) curve represents the mesons. The curves coincide for low $p_{\perp}$ value, i.e. the NCQ scaling is reproduced for the low $p_{\perp}$ region up to $\sim 1 \mathrm{GeV} / \mathrm{c}$. 
In [16] it is shown that the NCQ scaling is more precise if the $v_{2}$ coefficient is small. The three-cell model with one large stationary central cell and two moving side cells was also studied. The side cells were assumed to have the same velocities as in the case of the two-cell model, and the particle number ratio of the central cell to the side cells was set to $N_{c} / N_{s}=10$.

The temperature of all cells was the same. The obtained scaled $v_{2}\left(p_{\perp}\right)$ curves are shown in Fig. 5. The three-cell model is able to reproduce the NCQ scaling of $v_{2}$ for a wider range of $p_{\perp}$ values.

\section{Summary}

The model is simplified and attempts to provide an insight to the rapid hadronization and freeze out process in view of the NCQ scaling. We considered the breaking down of equilibrium in terms of thermo and fluid dynamical parameters, ot the different, $\mathrm{M}$ and $\mathrm{B}$, components of the matter. We intend to implement these concepts on the one hand in more complex models (like hybrid models) on the one side and to search for more fundamental reasons for the observed freeze out features.

This simple model is able of reproducing the NCQ scaling of the elliptic flow. The presence of NCQ scaling in experimental data suggests that the elliptic flow develops in the QGP phase, before the quarks recombine into hadrons. Therefore understanding the origin the elliptic flow can provide insight into the quark phase of matter.

\section{Acknowledgements}

Enlightening discussions with Prof. Daniel D. Strottman, Dr. Csaba Anderlik and Dr. Etele Molnar are gratefully acknowledged. This work was supported by the Alexander von Humboldt Foundation, by the Meltzer Fund of the University of Bergen and by the Computational Subatomic Physics Project at Uni-Research of the Research Council of Norway.

\section{References}

1. S. S. Adler et al. (PHENIX Collaboration), Phys. Rev. Lett. 91 (2003) 182301; J. Adams et al. (STAR Collaboration), Phys. Rev. Lett. 92 (2004) 052302.

2. P. Huovinen, P.F. Kolb, U. Heinz, P.V. Ruuskanen, S.A. Voloshin, Phys. Lett. B 503 (2001) 58

3. J. Cleymans, D. Elliott, A. Keranen, and E. Suhonen, Phys. Rev. C 57 (1998) 3319; J. Cleymans, H. Oeschler, and K. Redlich, Phys. Rev. C 59 (1999) 1663; A. Andronik, P. Braun-Munzinger, and J. Stachel, Nucl. Phys. A 772 (2006) 167; J. Cleymans, H. Oeschler, K. Redlich, and S. Wheaton, Acta Phys. Polonica B Proc. Suppl. 3 (2010) 533.

4. L. McLerran, and R.D. Pisarski, Nucl. Phys. A 796 (2007) 83.

5. I.N. Mishustin, L.M. Satarov, H. Stöcker, and W. Greiner, Phys. Atom. Nucl. 64 (2001) 802.

6. Hu Li, C.M. Shakin, Phys. Rev. D 66 (2002) 074016.

7. S. Zschocke, O.P. Pavlenko, B. Kämpfer, Eur. Phys. J. A 15 (2002) 529.

8. S. Zschocke, B. Kämpfer, O.P. Pavlenko, Gy. Wolf, arXiv:nucl-th/0202066.

9. S. Zschocke, and L.P. Csernai, Eur. Phys. J. A 39 (2009) 349.

10. L.P. Csernai, J.I. Kapusta, L.D. McLerran, Phys. Rev. Lett. 97 (2006) 152303-4.

11. Sz. Horvat, V.K.Magas, D.D. Strottman, and L.P. Csernai, Phys. Lett. B 692 (2010) 277.

12. S. Zschocke, Sz. Horvát, I. Mishustin, and L.P. Csernai, Submitted to Phys. Rec. C (2011).

13. T. Csörgő, L.P. Csernai, Phy. Lett. B 333 (1994) 494.

14. L.P. Csernai, and I.N. Mishustin, Phys. Rev. Lett. 74 (1995) 5005.

15. J. Randrup, Phys. Rev. C 82 (2010) 034902.

16. D. Molnar, Nucl. Phys. A 774 (2006) 257.

17. L.P. Csernai, Y. Cheng, V.K. Magas, I.N. Mishustin, and D. Strottman, Nucl. Phys. A 834 (2010) 261c. 